\title{
Power-Aware Provisioning of Virtual Machines for Real-Time Cloud Services
}

Kyong Hoon Kim ${ }^{1}$, Anton Beloglazov ${ }^{2}$, and Rajkumar Buyya ${ }^{2, *, \dagger}$

${ }^{1}$ Department of Informatics, Gyeongsang National University,

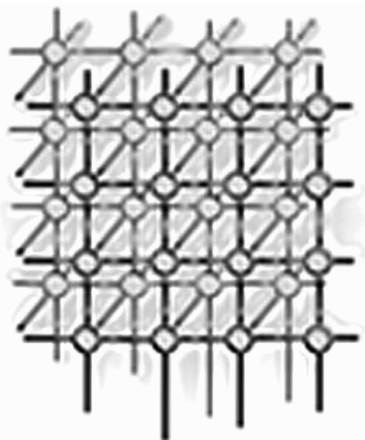
Gajwadong 900, Jinju, South Korea

${ }^{2}$ Cloud Computing and Distributed Systems (CLOUDS) Laboratory, Department of Computer Science and Software Engineering, The University of Melbourne, Parkville, Victoria 3010, Australia

\section{SUMMARY}

Reducing power consumption has been an essential requirement for Cloud resource providers not only to decrease operating costs, but also to improve the system reliability. As Cloud computing becomes emergent for the Anything as a Service (XaaS) paradigm, modern real-time services also become available through Cloud computing. In this work, we investigate power-aware provisioning of virtual machines for real-time services. Our approach is (i) to model a real-time service as a real-time virtual machine request; and (ii) to provision virtual machines in Cloud data centers using Dynamic Voltage Frequency Scaling (DVFS) schemes. We propose several schemes to reduce power consumption by hard real-time services and power-aware profitable provisioning of soft real-time services.

KEY WORDS: Cloud Computing, Real-Time Services, Energy-Efficient Computing, Green Data Centers

\section{Introduction}

Rapid developments in computing and communication technologies has led to the emergence of a new computing paradigm called Cloud computing, which delivers computing services to users as utilities in a pay-as-you-go manner [5]. Cloud providers offer various types of services, such as Infrastructure as a Service (IaaS), Platform as a Service (PaaS), and Software as a Service (SaaS). Service providers make use of IaaS and PaaS to deploy their services without concerns about management of physical resources. Under the Cloud computing model, users can access on-demand and pay-per-use services deployed anywhere in the world.

*Correspondence to: R. Buyya, CLOUDS Lab, The University of Melbourne, Parkville, Victoria 3010, Australia ${ }^{\dagger}$ E-mail: rbuyya@unimelb.edu.au 
Traditionally, enterprises purchased their own computing infrastructures to run real-time application, such as financial analysis, distributed data processing, real-time databases, etc. This approach provides performance guarantees, as the hardware is not shared and its capacity is provisioned to meet the needs of specific applications. However, owning an infrastructure incurs high operating costs and leads to the necessity to deal with the maintenance and upgrades of software and hardware. Moreover, this approach does not allow the infrastructure capacity to scale in and out depending on the current resource demand created by the applications. Cloud computing addresses these problems by providing dynamic resource provision on a pay-as-you-go basis. Cloud computing ensures performance requirements through Service Level Agreements (SLAs) negotiated between customers and Cloud providers. SLAs specify the agreements on the Quality of Service (QoS), such as deadline constraints. Thus, Cloud computing can be effectively leveraged as a platform for real-time applications.

One of the big challenges in data centers is to manage system resources in a power-efficient way. Data centers consume from 10 to 100 times more power per square foot than typical office buildings [23]. They can even consume as much electricity as a city [19]. The main part of power consumption in data centers comes from computation processing, disk storage, network, and cooling systems. Lowering the power usage of data centers becomes a challenging issue as computing applications and data are growing so quickly that increasingly larger servers and disks are needed to process them within the required time [6]. Thus, data center resources need to be managed in a power-efficient manner to drive Green Cloud computing [6]. In this paper, we study energy-efficient management of computing resources via Virtual Machine (VM) provisioning in Cloud computing environments.

To meet the growing demand for their services and ensure minimal costs, Cloud providers must implement power-efficient management of physical resources. Furthermore, as many applications require deadline constraints, power consumption in data centers must be minimized without violating the SLAs. This paper focuses on power-aware management of realtime Cloud services. The main contributions are: (i) a Cloud service framework for requesting a virtual platform for real-time applications; (ii) several power-aware VM provisioning schemes based on Dynamic Voltage Frequency Scaling (DVFS) for hard real-time services; and (iii) a power-aware profitable VM provisioning scheme for soft real-time services.

The remainder of this paper is organized as follows. Section 2 presents related work on power-aware Cloud computing. We propose a real-time Cloud service framework in Section 3. Section 4 describes the problem definition for hard real-time VM provisioning and provides several DVFS schemes. In Section 4, profitable VM provisioning of soft real-time services is analyzed based on DVFS schemes. We evaluate the proposed schemes using simulations in Section 6 , and conclude the paper with a summary and directions for the future work.

\section{Related Work}

Many of recent research works have focused on reducing power consumption in cluster systems. In $[1,31]$, high performance clusters with the consideration of power consumption have been designed and developed. As many recent commodity processors provide the DVFS capability, power-aware cluster systems have been built using such processors [13, 14]. Scientific applications developed based on the MPI library are mostly targeted at the reduction of 
power consumption $[15,13,25]$. Based on the profile of MPI programs, the authors choose an appropriate voltage scaling for each synchronization point.

General purpose cluster systems also have been studied on the reduction of power consumption. Srikantaiah et al. [26] have dealt with online services executing in heterogeneous clusters. When a new request comes, a heuristic for multidimensional bin packing is used to find a server to allocate the request. If a server cannot be found, a new machine is switched on and all the requests are re-allocated. Chase et al. [10] have aimed at serving web-applications in homogeneous clusters according to a utility function. Gandhi et al. [12] have investigated the problem of minimizing the mean response time of web-applications on heterogeneous clusters. In this work the optimal energy allocation is determined based on a theoretical queuing model.

The recently emerged Cloud computing paradigm leverages virtualization of computing resources and allows the achievement of more efficient allocation of the workload in terms of higher resource utilization and decreased power consumption [3]. Kusic et al. [18] have investigated the problem of minimizing both power consumption and SLA violations for online services in virtualized data centers using a limited look-ahead control. Verma et al. [28, 27] have proposed the pMapper architecture to solve the same problem considering the VM migration cost. Cardosa et al. [9] have presented several techniques for addressing the sharing-aware VM allocation problem. Hypervisor distributes resources among VMs according to a sharing-based mechanism, when the minimum and maximum amount of resources that can be allocated to a VM are specified.

In addition, many studies have focused on power-aware real-time applications in clusters. Rusu et al. [22] have developed a QoS-aware power management scheme by combining cluster-wide (On/ Off) and local (DVFS) power management techniques in the context of heterogeneous clusters. The front-end manager decides which servers should be turned on or off for a given system load, while the local manager reduces power consumption using DVFS. Wang et al. [30] have proposed a threshold-based method for efficient power management of heterogeneous soft real-time clusters as well as an offline mathematical analysis for determining the threshold. Kim et al. [17] have investigated power-aware algorithms for scheduling of realtime bag-of-tasks applications with deadline constraints in homogeneous clusters.

Considerable amount of work have been done in the area of power-efficient computing, but few of them deal with power-aware scheduling of real-time applications in Cloud computing environments. Buyya et al. [6] have presented their vision, challenges, and architectural elements for energy-efficient management of Cloud computing environments with consideration of QoS expectations. Kim et al. [16] have proposed a framework for provisioning Cloud resources for hard real-time services. In this paper, we extend our earlier work [16] in order to support general real-time services by considering the soft real-time model. Thus, this work investigates the problem of provisioning Cloud resources for both hard and soft real-time services in order to minimize power consumption. 


\section{Framework}

\subsection{Real-Time Service Model}

A usual real-time service such as financial analysis, distributed database, or image processing, consists of multiple real-time applications or subtasks. As long as a group of applications for a given real-time service meet all their deadlines, the service accomplishes the QoS agreed with users. A real-time service is defined by $\left\{\tau_{i}\left(r_{i}, c_{i}, d_{i}, p_{i}, f_{i}\right) \mid i=1, \ldots, n\right\}$, where $n$ is the number of subtasks. Each real-time subtask $\tau_{i}$ is defined by the following parameters.

- $r_{i}$ : release time

- $c_{i}$ : worst-case execution time

- $d_{i}$ : relative deadline

- $p_{i}$ : period

- $f_{i}$ : finish time

A real-time subtask $\tau_{i}$ can be started at the time $r_{i}$ and requires the worst-case execution time $c_{i}$. In order to accomplish the application's objective, it should be completed by the time $r_{i}+d_{i}$. Also, $p_{i}$ specifies its periodicity so that the application releases a subtask of $c_{i}$ computation time at the time $\left(r_{i}+k p_{i}\right)$, and should be finished by $r_{i}+k p_{i}+d_{i}(k=0,1, \ldots)$. In case of a non-periodic application, $p_{i}$ is set to zero. We also consider the duration or finish time, $f_{i}$, since a user cannot have access to a Cloud computing resource forever, although a periodic real-time task in an embedded system assumes an infinite sequence.

A group of sub-tasks of a real-time service is developed and launched on a specific runtime platform including middleware, operating system, etc. A Cloud computing environment is a suitable solution for real-time services, as it leverages virtualization. When users request execution of their real-time services in a Cloud computing environment, appropriate VMs are provisioned for those services.

\subsection{Real-Time Virtual Machine Model}

Regarding the deadline model, real-time services can be categorized into two types: hard and soft. In the hard deadline model, a service provider receives some penalty if a service does not meet the deadline. On the other hand, a service with a soft deadline provides a diminished value or utility even when the execution time exceeds the deadline. A penalty function, such as linear decreasing function, is used in the soft deadline model to define the value decrease. Thus, we propose two different real-time VM models for Cloud computing.

\subsubsection{Hard Real-Time Virtual Machine Model}

In this subsection, we define HRT-VM (Hard Real-Time Virtual Machine) as a requirement for a VM providing a hard real-time service. HRT-VM $V_{i}$ for a hard real-time service is described by three parameters: $u_{i}, m_{i}$, and $d_{i}$.

- $u_{i}$ : the CPU utilization required for the real-time application 


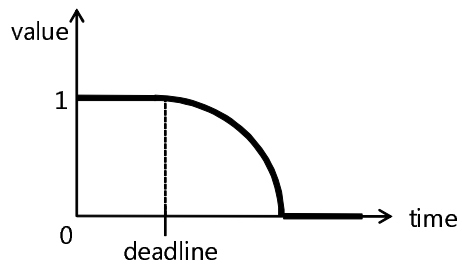

(a) Concave penalty function

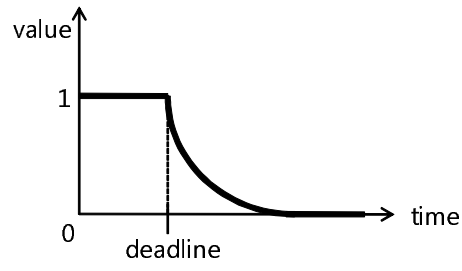

(b) Convex penalty function

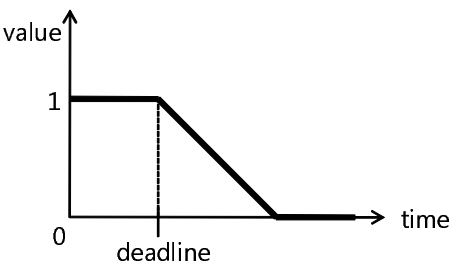

(c) Linear penalty function

Figure 1. Various soft real-time service models

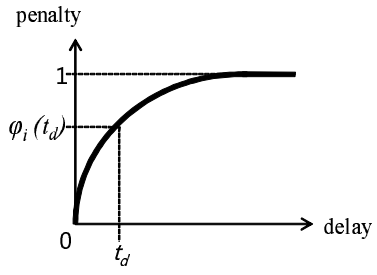

(a) Penalty function

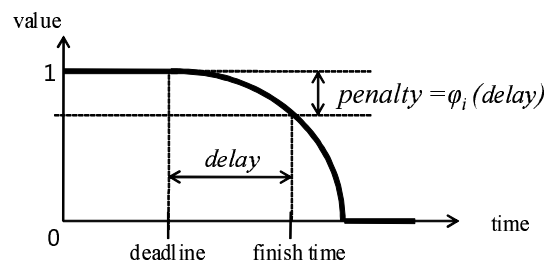

(b) Service value function

Figure 2. Quality of soft real-time service

- $m_{i}$ : the number of Millions Instructions Per Second (MIPS) required for the base VM

- $d_{i}$ : the lifetime or deadline

The service is developed and launched on a specific platform or infrastructure (e.g. 1GHzLinux machine). We select the MIPS rate, $m_{i}$, for the specification of the base VM. For a given set of real-time applications, we can analyze the required CPU utilization $u_{i}$ on the base VM. Thus, the above requirement implies that the real-time service is guaranteed when the allocated VM keeps providing $u_{i} \times m_{i}$ of the processing capacity by the deadline $d_{i}$. This realtime service on a virtualized Cloud resource is achieved by compositional real-time computing and real-time $V M$ techniques.

\subsubsection{Soft Real-Time Virtual Machine Model}

The requirement for a VM providing a soft real-time service includes an additional parameter, called the penalty function. The penalty function indicates the diminished value of a service by executing a VM that has missed the deadline. Figure 1 shows examples of soft real-time service models with various penalty functions. If a real-time service meets its deadline, it provides the pre-determined value. However, when it misses the deadline, the value or utility of the service decreases according to its penalty function.

$$
\text { value }= \begin{cases}1 & \text { if delay } \leq 0 \\ \varphi_{i}(\text { delay }) & \text { if delay }>0 .\end{cases}
$$


We refer to the model of a soft real-time VM as SRT-VM (Soft Real-Time Virtual Machine). SRT-VM $V_{i}$ for a soft real-time service is defined by $\left(u_{i}, m_{i}, d_{i}, \varphi_{i}\right)$, where $\varphi_{i}$ denotes the penalty function of the soft real-time service model. If the $\mathrm{VM} V_{i}$ is provided with $u_{i} \times m_{i}$ of processing capacity by the deadline $d_{i}$, the service quality is guaranteed. However, if the processing capacity of $u_{i} \times m_{i}$ requires the time beyond the deadline, the service quality is defined by the penalty function $\varphi_{i}$. Let us assume that $V_{i}$ is provided with the capacity past delay time units. Then, as shown in Figure 2, the service quality or value is given by Equation (1).

\subsubsection{Real-Time Virtual Machine Request}

Cloud resource brokers take the role of finding Cloud resources or VMs for real-time services requested by users. Thus, a user requests VMs by either HRT-VM or SRT-VM depending on deadline types, as described above. We denote a real-time VM request as $R T$-VM regardless of the deadline type.

The compositional or hierarchical real-time framework [11, 24] enables a group of realtime applications to be a single real-time resource requirement to the upper layer of a realtime environment. Thus, we assume that the RT-VM $V_{i}$ is defined by multiple real-time applications, $\left\{\tau_{k}\left(r_{k}, w_{k}, d_{k}, p_{k}, f_{k}\right) \mid k=1, \ldots, n\right\}$, of the service by using the compositional real-time technique. Thus, the VM provisioner in a Cloud allocates VMs for services rather than for individual applications. Furthermore, recent work on implementing real-time VMs [29, 32] assures real-time services (e.g. real-time CPU allocation, real-time I/O) of a VM. This paper focuses on how to provision VMs to a given RT-VM request with the consideration of power consumption by leveraging the described techniques.

\subsection{Real-Time Cloud Service Framework}

In this subsection, we describe the real-time Cloud service framework based on the real-time VM model. As shown in Figure 3, the steps taken by a user to execute a real-time service are as follows.

(1) Requesting a virtual platform: A user who wants to launch a real-time service submits all the information about the real-time applications to the broker.

(2) Generating a RT-VM from real-time applications: The resource broker first analyzes the submitted real-time applications and generates one RT-VM request, $V_{i}=\left(u_{i}, m_{i}, d_{i}\right)$.

(3) Requesting a real-time VM: The broker requests a VM for RT-VM $V_{i}$ from the VM provisioner of a Cloud computing environment.

(4) Mapping physical processors: The VM provisioner finds appropriate processing elements that meet the $V_{i}$ requirements and provides the VM to the user.

(5) Executing the real-time applications: The user launches and executes the real-time applications using the provided VM. 


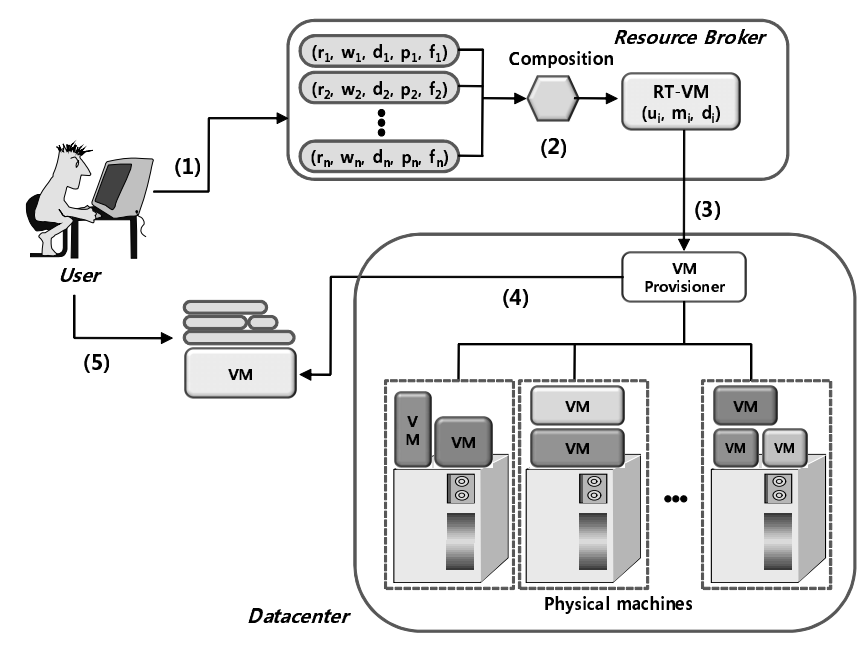

Figure 3. Framework

\section{Power Model}

The main part of power consumption in data centers comes from computation processing, disk storage, network, and cooling systems. This paper focuses on reduction of CPU power consumption using power-aware VM provisioning in Cloud computing environments.

The most of power consumption in CMOS circuits is composed of dynamic and static power. We only consider the dynamic power consumption, as it is the dominating factor in the total power consumption and can be managed using Dynamic Power Management Techniques (DPM) [20]. Cloud providers can increase their profit by reducing the dynamic power consumption. The dynamic power consumption by a CPU is proportional to $V_{d d}^{2}$ and $f$, where $V_{d d}$ is the supply voltage and $f$ is the frequency [4]. Since the frequency is usually in proportion to the supply voltage, the dynamic power consumption of a processor is defined in Equation (2).

$$
P=C \cdot f^{3},
$$

where $C$ is a coefficient of proportionality. Let us consider an application with the execution time $t$ running at a CPU with the frequency $f_{\max }$. If the processor runs at the frequency level $f\left(0<f \leq f_{\max }\right)$, the execution time is defined by $t / \frac{f}{f_{\max }}$. Thus, the dynamic power consumption during the task execution is defined as in Equation (3).

$$
\begin{aligned}
E & =\int_{0}^{t / \frac{f}{f_{\max }}} P=C \cdot t \cdot f_{\max } \cdot f^{2} \\
& =\alpha \cdot t \cdot S^{2}
\end{aligned}
$$




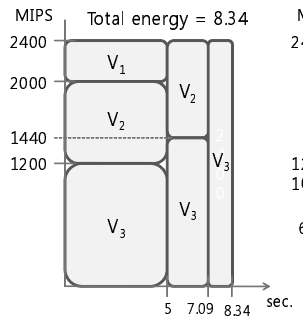

(a) Maximum Speed

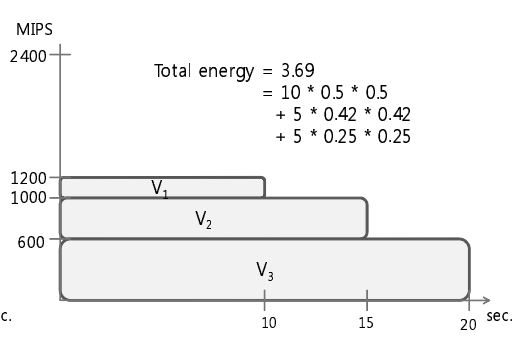

(b) DVS

Figure 4. Proportional sharing of VM provisioning and power consumption $(\alpha=1)$

where $\alpha$ is a coefficient and $S$ is the relative processor speed for the frequency $f$ ( $S=$ $f\left(f_{\max }\right)$. The DVFS scheme reduces the dynamic power consumption by decreasing the supplying voltage and frequency, which results in a slowdown of the CPU and increased execution time. We assume that each PE (Processing Element) $p$ in a datacenter can adjust its processor frequency from $f_{p}^{\min }$ to $f_{p}^{\max }$ continuously. The relative processor speed $S$ for each frequency $f$ is defined by $f / f_{\max }$, where $f_{p}^{\min } / f_{p}^{\max }<S \leq 1$.

\section{Power-Aware Hard Real-Time Cloud Service}

\subsection{Problem Description}

Let us consider a physical machine with one PE of 2400 MIPS and a set of HRT-VMs, $\left\{V_{1}(0.2,1000,10), V_{2}(0.8,500,15), V_{3}(0.5,1200,20)\right\}$, as an example. $V_{1}$ requires the utilization $20 \%$ on 1000-MIPS machine by the deadline 10 sec. Similarly, $V_{2}$ and $V_{3}$ require $80 \%$ and $50 \%$ of 500-MIPS and 1200-MIPS machines by 15 and 20 seconds respectively. Figure 4(a) shows the proportional sharing scheduling result of these VMs under the maximum processor capacity. The proportional share of $V_{i}$ is defined by $m_{i} \times u_{i} / \sum_{j=1}^{3}\left(m_{j} \times u_{j}\right)$. Three HRT-VMs share the processor capacity in proportion to their required MIPS rates, $m_{i} \times u_{i}$, and finish before the deadlines. The total power consumption is $8.34\left(=1 \times 8.34 \times 1.0^{2}\right)$ according to Equation (3) under the assumption of $\alpha=1$.

The power consumption can be reduced by combining DVFS and the proportional sharing scheduling. As shown in Figure 4(b), the minimum required processor capacity is allocated to each VM, so that the processor dynamically adjust its speed to $\sum\left(m_{j} \times u_{j}\right) / 2400$. The total power consumption of the DVFS scheme is $3.69\left(=10 \times 0.5^{2}+5 \times 0.42^{2}+5 \times 0.25^{2}\right)$. Thus, the DVFS scheme can significantly reduce power consumption compared to the maximum-speed static scheme.

However, there are trade-offs in the dynamic scaling of the processor speed in on-line realtime Cloud computing. Operation at a higher processor speed allows the acceptance of more HRT-VMs but leads to higher power consumption. On the contrary, scaling the processor speed 
Table I. Remaining service times of Figure 4(a)

\begin{tabular}{|c|c|c|c|c|c|c|c|c|c|c|}
\hline & $t=0$ & \multicolumn{3}{|c|}{$t=5$} & \multicolumn{3}{|c|}{$t=7.09$} & \multicolumn{3}{|c|}{$t=8.34$} \\
\hline & $w_{i}$ & $\begin{array}{c}Q_{i} \\
{[0,5]}\end{array}$ & $\begin{array}{c}S T_{i} \\
{[0,5]}\end{array}$ & $w_{i}$ & $\begin{array}{c}Q_{i} \\
{[5,7.09]}\end{array}$ & $\begin{array}{c}S T_{i} \\
{[5,7.09]}\end{array}$ & $w_{i}$ & $\begin{array}{c}Q_{i} \\
{[7.09,8.34]}\end{array}$ & $\begin{array}{c}S T_{i} \\
{[7.09,8.34]}\end{array}$ & $w_{i}$ \\
\hline$V_{1}$ & 2000 & 400 & 2000 & 0 & - & - & - & - & - & - \\
\hline$V_{2}$ & 6000 & 800 & 4000 & 2000 & 960 & 2000 & 0 & - & - & - \\
\hline$V_{3}$ & 12000 & 1200 & 6000 & 6000 & 1440 & 3010 & 2990 & 2400 & 2990 & 0 \\
\hline
\end{tabular}

down results in lower consumed power with lower acceptance. For example, let us assume that a new HRT-VM $V_{4}(0.8,2000,10)$ is requested at the time 10 . Figure $4(\mathrm{a})$ accepts $V_{4}$ since the processor is idle at the time 10, while the DVFS scheme shown in Figure 4(b) cannot provision it due to lack of the processor capacity.

Data centers can increase their profit by provisioning more VMs to users. However, reducing power consumption increases the profit by reducing the operating cost. To address this tradeoff, we propose several schemes for power-aware provisioning of real-time VMs for the purpose of maximizing profits in Cloud data centers.

We use the proportional sharing scheduling to schedule multiple VMs on a processor. The proportional sharing scheduling is simple but guarantees the real-time services of HRT-VMs if the total required MIPS rate is less than or equal to the processor capacity. Furthermore, it can be easily implemented. For example, the default scheduling in Xen Hypervisor [21] is Credit scheduler which is based on credit value set by weight of each VM. The VMM (Virtual Machine Monitor) can dynamically adjust credit values of VMs according to their required MIPS rates in order to support the proportional sharing scheme.

Before explaining the VM provisioning, we define the remaining service time, $w_{i}$, of $V_{i}$. The initial value of $w_{i}$ is defined by $u_{i} \times m_{i} \times\left(d_{i}-t_{s}\right)$, at its submission time $t_{s}$. If $V_{i}$ is provided with $q_{i}$ MIPS rate for the period $t_{p}, w_{i}$ is decreased by $q_{i} \times t_{p}$. For instance, Table I shows the remaining service times of the three described HRT-VMs at the time of proportional share change shown in Figure 4(a). $V_{i}$ finishes its service when $w_{i}$ becomes zero.

\subsection{DVFS-Enabled HRT-VM Provisioning}

When a data center receives a HRT-VM request from a resource broker, it returns the price of providing the HRT-VM service if it can provide real-time VMs for that request. The broker selects the minimally priced VM among available data centers. Thus, the provisioning policy in this paper is to select the processing element with the minimum price for the sake of users. Figure 5 shows the pseudo-code of the algorithm for provisioning a VM for a HRT-VM request.

For a given HRT-VM $V_{i}\left(u_{i}, m_{i}, d_{i}\right)$, the data center checks the schedulability of $V_{i}$ on the processing element $P E_{k}$ of $Q_{k}$ MIPS rate. Suppose that the current running HRT-VMs on the processing element $P E_{k}$ at time $t$ is known as $T_{k}=\left\{V_{j}\left(u_{j}, m_{j}, d_{j}\right) \mid j=1, \cdots, n_{k}\right\}$. And the remaining service time of $V_{j}$ at the time $t$ is denoted as $w_{j}$. Then, the schedulability is 


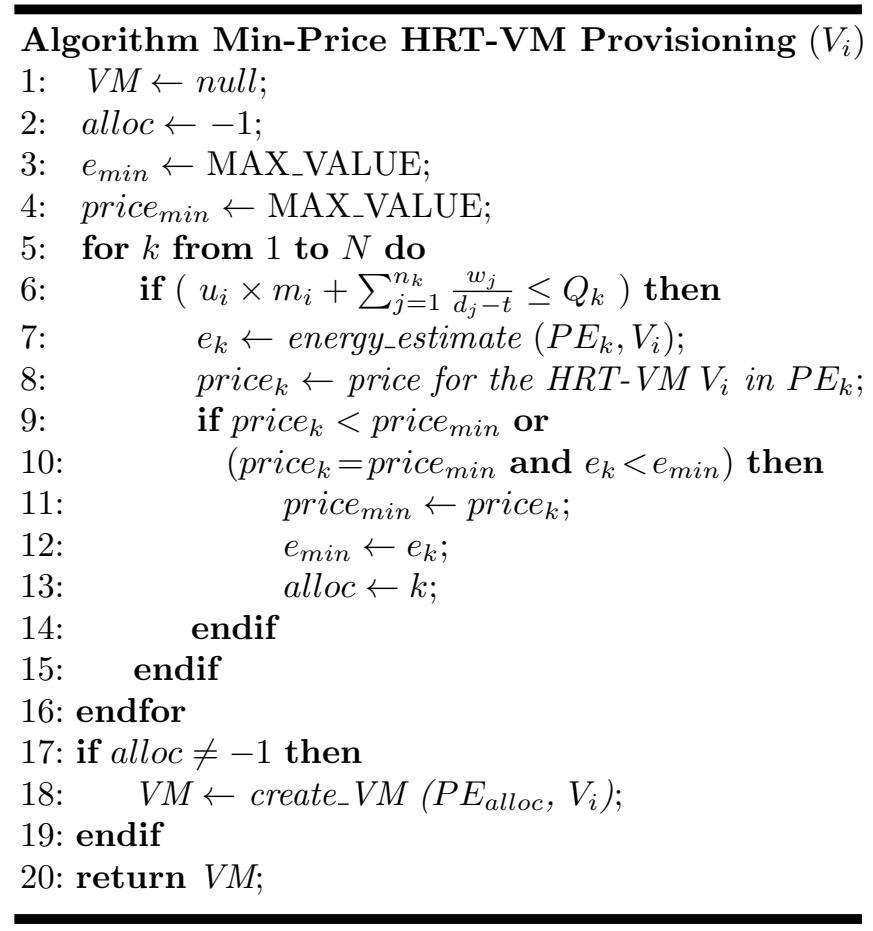

Figure 5. Min-price HRT-VM provisioning

guaranteed if it satisfies Equation (4). Since $w_{j} /\left(d_{j}-t\right)$ is the minimum MIPS rate for $V_{j}$ by its deadline $d_{j}$, Equation (4) means that the total summation of all the required MIPS rates including the new HRT-VM $V_{i}$ is less than the processor capacity $Q_{k}$.

$$
u_{i} \times m_{i}+\sum_{j=1}^{n_{k}} \frac{w_{j}}{d_{j}-t} \leq Q_{k}
$$

If $P E_{k}$ is able to schedule $V_{i}$, it estimates the energy and price of provisioning (line 7 , 8). Since the provisioning policy is to provide a lower price to users, the algorithm finds the minimally priced processor. For the same price, less energy is preferable because it produces higher profit (line 9-14). Finally, a VM is mapped on $P E_{a l l o c}$ if HRT-VM $V_{i}$ is schedulable in the data center.

When a user launches the service on the VM, the resource provider provisions the VM using DVFS schemes to reduce power consumption. We propose three power-aware VM provisioning schemes: Lowest-DVFS, $\delta$-Advanced-DVFS, and Adaptive-DVFS. The following subsections describe them. 


\subsubsection{Lowest-DVFS for VM Provisioning}

This scheme adjusts the processor speed to the lowest level at which HRT-VMs meet their deadlines. That is, each HRT-VM $V_{i}$ executes its service at the required MIPS rate, as shown in Figure 4(b). It consumes the lowest energy in the case when the HRT-VM arrival rate is low enough to accept all the requests.

\subsection{2. $\delta$-Advanced-DVFS for VM Provisioning}

In order to overcome the low service acceptance rate of Lowest-DVFS scheme, this scheme over-scales up to $\delta \%$ of the required MIPS rate for current HRT-VMs. Thus, it operates the processor $\delta \%$ faster in order to increase the possibility of accepting incoming HRT-VM requests. The processor scale $s$ is adjusted as in Equation (5) at the time $t$ for a given HRT-VM set $T_{k}$. The proportional share of each VM is in proportion to $w_{i} /\left(d_{i}-t\right)$.

$$
s=\min \left\{1,\left(1+\frac{\delta}{100}\right) \times \frac{1}{Q_{k}} \sum_{V i \in T_{k}} \frac{w_{i}}{d_{i}-t}\right\}
$$

The value of $\delta \%$ is predefined in the system according to the system load. Throughout the simulation results in Section 5, we analyze the impact of $\delta$.

\subsubsection{Adaptive-DVFS for VM Provisioning}

When the HRT-VM arrival rate and their service times are known in advance, we can analyze the optimal scale. Let us consider the $M / M / 1$ queuing model with the arrival rate $\lambda$ and service rate $\mu$. If the processor speed scale is set to $s$, then the average response time, $R T$, is given by $R T=1 /(s \mu-\lambda)$, according to the $M / M / 1$ queuing model. In addition, the response time should be less than or equal to the average deadline, $d$, in order to meet the real-time service requirements $(1 /(s \mu-\lambda) \leq d)$. Thus, the optimal scale, $s^{*}$, to reduce the power consumption is given by Equation (6).

$$
s^{*}=\frac{1}{\mu}\left(\lambda+\frac{1}{d}\right)
$$

Adaptive-DVFS scheme manages the average arrival rate $\hat{\lambda}$, the average service rate $\hat{\mu}$, and the average deadline $\hat{d}$ for the last $h$ service requests (e.g. $h=10)$. It adjusts the processor scale $s$ as in Equation (7) at time $t$ for a given HRT-VM set $T_{k}$.

$$
s=\max \left\{\min \left\{1, \frac{1}{\hat{\mu}}\left(\hat{\lambda}+\frac{1}{\hat{d}}\right)\right\}, \frac{1}{Q_{k}} \sum_{V i \in T_{k}} \frac{w_{i}}{d_{i}-t}\right\}
$$

In Equation (7), the optimal scale is calculated by Equation (6) not greater than one. Since it should be greater than the minimum required utilization of the current HRT-VMs on the processor, we select the maximum between the two values. The processor speed is adjusted according to Equation (7) when a new HRT-VM is provided or an existing one finishes its execution. 


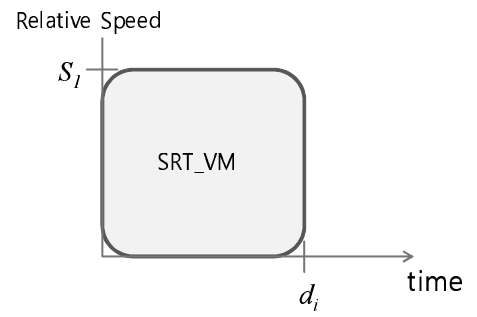

(a) Meeting deadline

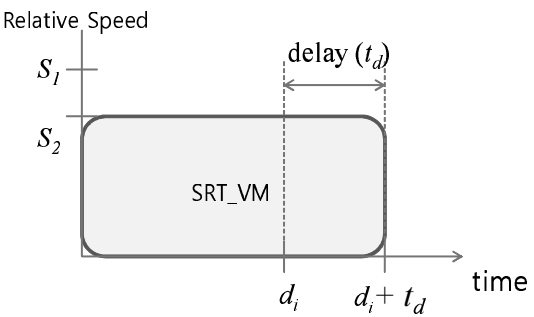

(b) Missing deadline

Figure 6. DVFS-based SRT-VM provisioning

\section{Power-Aware Soft Real-Time Cloud Service}

\subsection{Profitable Delay Analysis}

When a soft real-time service misses its deadline, it gives a diminished value of the service to the user. In this case, the user does not need to pay the whole price for the Cloud resources because the resource provider has not met the QoS requirements. In this paper, we assume that a refund due to a service delay is in proportion to the diminished value of the service. The profit of a Cloud resource provider for providing a soft real-time service $i$ with consideration of a refund is defined by Equation (8).

$$
\begin{aligned}
\text { profit }_{i} & =\text { price }_{i} \times \text { value }_{i}-\text { cost }_{i} \\
& =\text { price }_{i} \times\left(1-\varphi_{i}\left(\text { delay }_{i}\right)\right)-\operatorname{cost}_{i},
\end{aligned}
$$

where value $_{i}$ is the service quality of the soft real-time service depending on the finish time, as shown in Figure 2; and cost $_{i}$ is the total cost including the power consumption.

Since a soft real-time service gives a value beyond the deadline, the power-aware provisioning may produce more profit in case of delayed service execution. Figure 6(a) shows the case of the highest profit when the deadline is met, as in Figure 4. Let us assume that the cost of $t$ execution time is in proportion to the power consumption as in Equation (3). Then, the cost function of $t$ execution time is defined by Equation (9).

$$
\operatorname{cost}(t)=\beta \cdot t \cdot S^{2}
$$

where $\beta$ is a coefficient and $S$ is the associated relative processor speed.

Let us assume that the penalty function of a SRT-VM $i$ is given by a linear function with the penalty rate $k$, as shown in Figure 1(c). Then, the profit of finishing at the time $d_{i}+t_{d}$, as shown in Figure 6(b), is given by Equation (10).

$$
\operatorname{profit}_{i}\left(d_{i}+t_{d}\right)=\text { price }_{i} \times\left(1-k t_{d}\right)-\beta\left(d_{i}+t_{d}\right) S_{2}^{2} .
$$




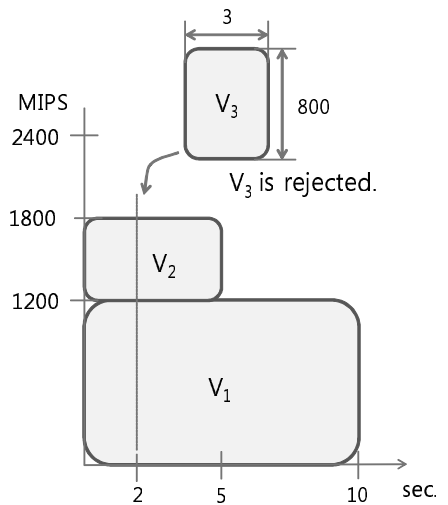

(a) DVFS based on HRT-VM

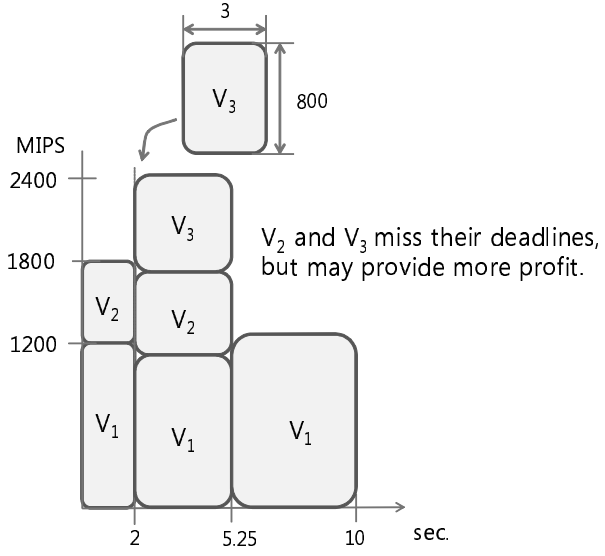

(b) DVFS based on SRT-VM

Figure 7. Acceptance of a RT-VM in HRT-VM and SRT-VM models

Thus, the condition that the profit of delaying the service of the SRT-VM $i$ is greater than that of meeting the deadline is given by Equation (11). That is, the case of Figure 6(b) may provide more profit to the resource provider than the case of Figure $6(\mathrm{a})$, if the delayed service time, $t_{d}$, satisfies Equation (11).

$$
\text { price }_{i} \times\left(1-k t_{d}\right)-\beta\left(d_{i}+t_{d}\right) S_{2}^{2}>\text { price }_{i}-\beta d_{i} S_{1}^{2}
$$

The inequality of Equation (11) is shown in terms of the delayed service time, as in Equation (12).

$$
t_{d}<\frac{S_{1}^{2}-S_{2}^{2}}{\text { price }_{i} k+\beta S_{2}^{2}}
$$

\subsection{Resource Provisioning of SRT-VM}

When an SRT-VM request is received, a data center provides for the resource broker an appropriate VM. Similarly to the HRT-VM provisioning algorithm in Figure 5, a data center finds for the user the minimally priced resource. The difference from the HRT-VM provisioning is the acceptance test of a VM. In Figure 5, a new VM request is accepted if all VMs on a $\mathrm{PE}$ including the new one meet their deadlines, as in Equation (4). However, the SRT-VM provisioning algorithm accepts a new VM request as long as the acceptance results in higher profit. For example, let us consider two VM requests $V_{1}$ and $V_{2}$ accepted at the time 0 on a PE, as shown in Figure $7(\mathrm{a})$. When a new VM $V_{3}$ requiring 800 MIPS rate during 3 time units arrives at the $\mathrm{PE}$, the HRT-VM provisioning algorithm rejects the request since it cannot 


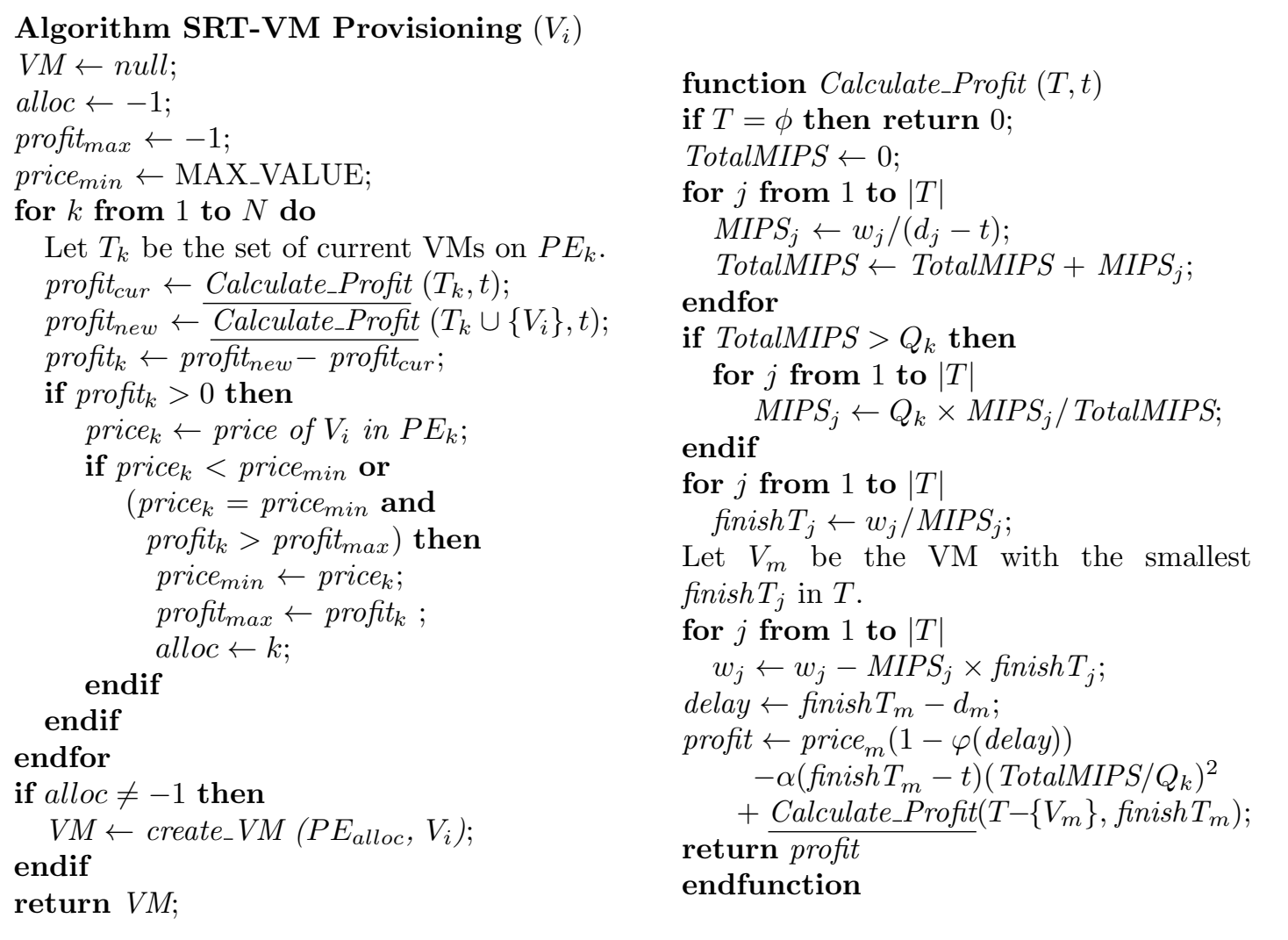

Figure 8. Min-price profitable SRT-VM provisioning

meet the deadline. On the contrary, SRT-VM can accept $V_{3}$ if the acceptance produces higher profit, as shown in Figure 7(b).

Therefore, the provisioning of SRT-VM should consider the profit in the acceptance test. Figure 8 shows the pseudo-code of the algorithm for the SRT-VM provisioning. The left side of Figure 8 describes the minimally priced VM allocation, in which it calculates the profit by calling the function Calculate_Profit (). The function Calculate_Profit () is shown in the right side of Figure 8 for a given set of VM-request at the time $t$. If the number of VMs in $T$ is $n_{k}$, there may be $n_{k}$ different finish times. The function calculates the profit for each finish time by adding the VM profit minus the cost. The function Calculate_Profit () implements this by calling the function recursively without deleting the earliest finishing VM, and adding 


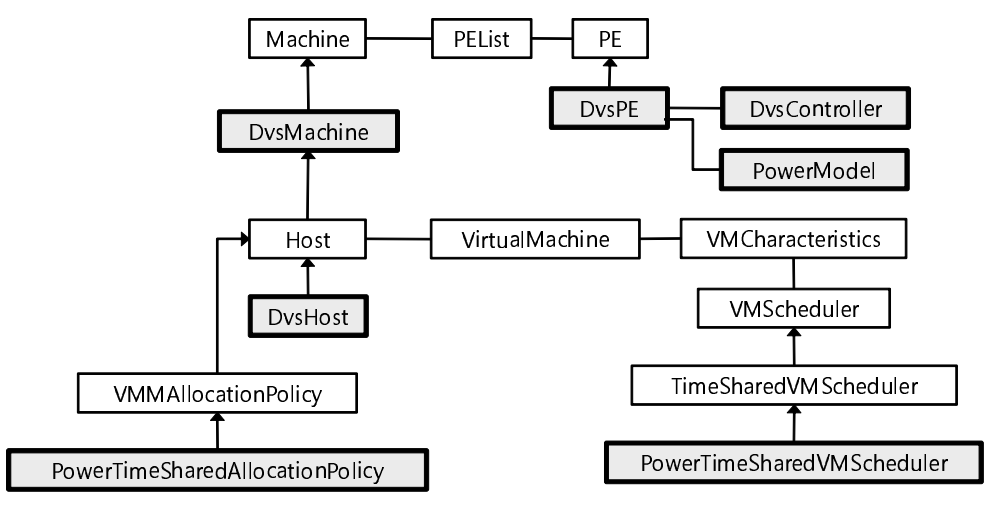

Figure 9. CloudSim architecture

Table II. Characteristics of the data center

\begin{tabular}{|c|c|c|c|c|}
\hline & \# of PEs & MIPS of PE & DVFS level & $\alpha\left(10^{-3}\right)$ \\
\hline Machine 0 & 4 & 1,800 & {$[0,1.0]$} & 2.92 \\
\hline Machine 1 & 4 & 2,400 & {$[0,1.0]$} & 4.08 \\
\hline Machine 2 & 4 & 3,000 & {$[0,1.0]$} & 5.37 \\
\hline Machine 3 & 4 & 3,400 & {$[0,1.0]$} & 6.21 \\
\hline
\end{tabular}

the returned profit. The time complexity of the profit calculation is $O\left(n_{k}^{2}\right)$, where $n_{k}$ is the number of VMs on a PE.

\section{Simulation Results}

We have evaluated the proposed algorithms by simulations of power-aware real-time services using the CloudSim toolkit [7, 8] with an extension enabling power-aware simulations. In order to support the power-aware VM provisioning, we have implemented additional components for the CloudSim toolkit, as shown in Figure 9. DvsPE, DvsMachine, and DvsHost are DVFS-enabled PE, machine, and host, respectively. The component PowerTimeSharedAllocationPolicy inherits the generic VM provisioning class and implements the simulated algorithms. The local scheduler in a VM is implemented by PowerTimeSharedVMScheduler.

We have created a data center with four machines with 16 DVFS-enabled processors with the characteristics shown in Table II. The price model in the simulations follows the Amazon EC2 Standard small (default) instance type [2], so that the unit price per hour equals to $\$ 0.10$. We use the cost function as the power consumption of each machine in Table II. 


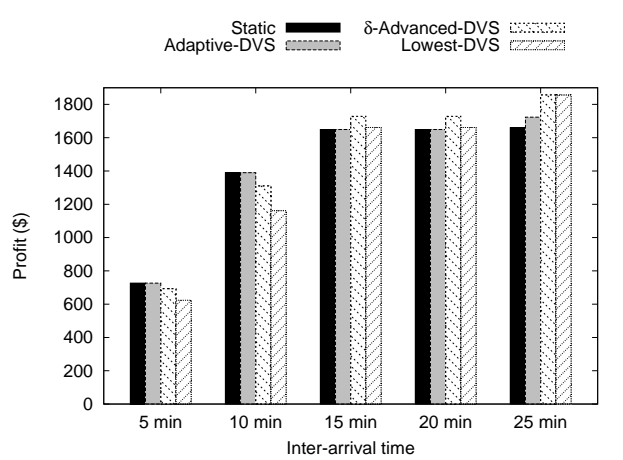

(a) Total profit

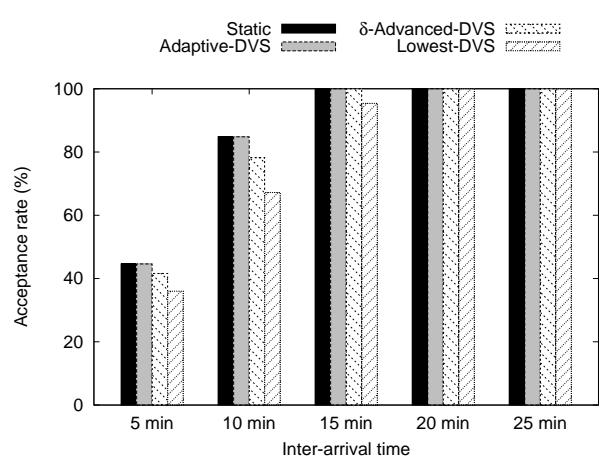

(b) Acceptance rate

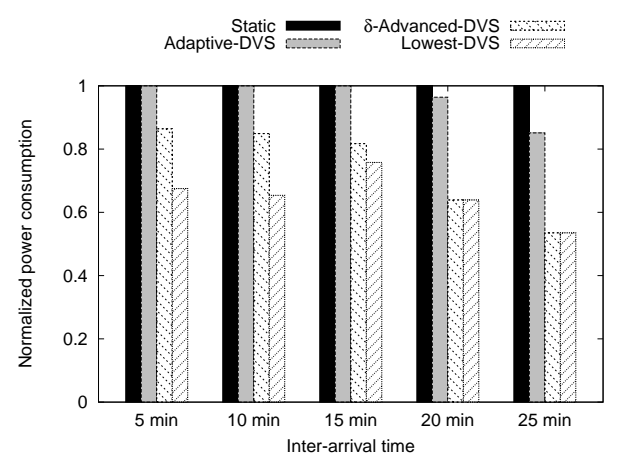

(c) Normalized power consumption

Figure 10. Simulation results

In the simulations, we have generated 500 HRT-VMs. The total service length $\left(w_{i}\right)$ of a HRT-VM is randomly selected from 2,400 GIs $\left(10^{3} \mathrm{MIs}\right)$ to 3,600 GIs. The deadline is selected from 10 to 30 minutes more than the execution time based on a 1000-MIPS machine. The interarrival time between two HRT-VMs follows the Poisson distribution. We have simulated various interarrival times.

Figure 10(a) shows the total profits generated by each scheme according to the interarrival time. Static does not use DVFS so that it runs VMs at the maximum processor speed. In $\delta$-Advanced-DVFS we fix $\delta$ as $15 \%$. For higher arrival rates, Static produces higher profits since it accepts more HRT-VMs. Adaptive-DVFS produces not less profit than Static, while other DVFS schemes generate higher profit for lower arrival rates due to lower power consumption.

Figure 10 (b) and (c) show the HRT-VM acceptance rate and the normalized power consumption compare to Static, respectively. The acceptance rate of Adaptive-DVFS is close to Static but reduces much energy in case of a low arrival rate. $\delta$-Advanced-DVFS shows a higher acceptance rate with similar power consumption compared to Lowest-DVFS. Generally, $\delta$-Advanced-DVFS shows the best performance in terms of the profit per unit of 


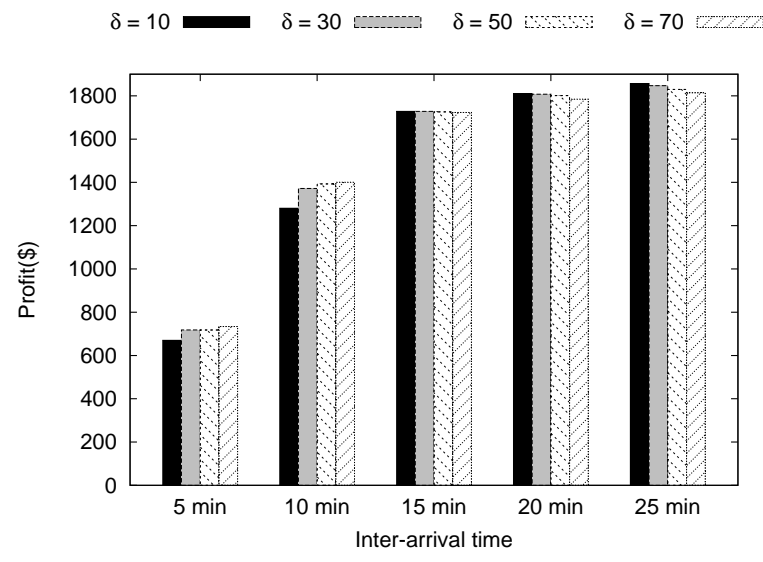

Figure 11. Impact of $\delta$ in $\delta$-Advanced-DVFS

the consumed power since the amount of scaling up is controlled automatically according to the system load. In the case of Adaptive-DVFS, its performance is limited by the simplified queueing model.

Next, we have varied the value of $\delta$ in order to analyze its impact. As shown in Figure 11, higher $\delta$ show better performance for higher arrival rates, since it leads to more VMs being accepted. On the contrary, lower $\delta$ produce higher profit in case of lower arrival rates. In the simulations, the system utilization is generally high regardless of the arrival rate; therefore, $\delta$ has a little impact on the profit.

\section{Conclusion and Future Work}

In this paper, we have proposed a real-time Cloud service framework where each real-time service request is modeled as RT-VM in resource brokers. We have investigated poweraware provisioning of VMs for soft and hard real-time Cloud services. For hard real-time services, we have provided several schemes and evaluated them using simulations. For soft real-time services, we have analyzed power-aware profitable VM provisioning and proposed a provisioning algorithm. The simulation results have shown that data centers can reduce power consumption and increase their profit using DVFS schemes. The proposed adaptive schemes, Adaptive-DVFS and $\delta$-Advanced-DVFS, produce higher profit with lower power consumption regardless of the system load.

Our on-going work includes further analysis and improvement of the proposed adaptive schemes. For example, $\delta$ in $\delta$-Advanced-DVFS can be adjusted depending on the system load, or Adaptive-DVFS can be modified using other queueing models rather than $M / M / 1$. Moreover, we will compare the proposed schemes with other approaches, such as bin packing and linear programming, and analyze the impact of the cooling systems. We also plan to deeper investigate 
the soft real-time VM provisioning with the consideration of various penalty functions. Once the investigation is completed, we plan to implement the proposed framework in a Cloud broker and run real experiments to validate the practical applicability of the approach.

\section{Acknowledgements}

This is an extended version of the paper presented at the MGC 2009 Workshop [16]. This work was primarily carried out during the first author's visit to the CLOUDS Lab at the University of Melbourne. We thank external reviewers and the Guest Editor of this special issue for their suggestions on enhancing the quality of the paper.

\section{REFERENCES}

1. Adiga ND, et al. An overview of the BlueGene/L supercomputer. In Proceedings of the ACM/IEEE Supercomputing Conference. Baltimore, USA, November 2002.

2. Amazon Elastic Compute Cloud (Amazon EC2). http://aws.amazon.com/ec2.

3. Beloglazov A, Buyya R, Lee YC, Zomaya A. A Taxonomy and Survey of Energy-Efficient Data Centers and Cloud Computing Systems. Advances in Computers, M. Zelkowitz (editor), ISBN 13: 978-0-12-012141-0, Elsevier, 2011.

4. Burd TD, Brodersen RW. Energy efficient cmos microprocessor design. In Proceedings of the 28th Annual Hawaii International Conference on System Sciences. Kihei, Maui, Hawaii, USA, January 1995.

5. Buyya R, Yeo CS, Venugopal S, Broberg J, Brandic I. Cloud computing and emerging IT platforms: vision, hype, and reality for delivering computing as the 5th utility. Future Generation Computer Systems. 25(6):599-616, Elsevier Science, Amsterdam, The Netherlands, June 2009.

6. Buyya R, Beloglazov A, Abawajy J. Energy-efficient management of data center resources for cloud computing: a vision, architectural elements, and open challenges. In Proceedings of the 2010 International Conference on Parallel and Distributed Processing Techniques and Applications (PDPTA 2010). Las Vegas, USA, July 2010.

7. Buyya R, Ranjan R, Calheiros RN. Modeling and simulation of scalable Cloud computing environments and the CloudSim toolkit: Challenges and opportunities. In Proceedings of the 7th High Performance Computing and Simulation (HPCS 2009). Leipzig, Germany, June 2009.

8. Calheiros RN, Ranjan R, Beloglazov A, De Rose CAF, and Buyya R. CloudSim: A Toolkit for Modeling and Simulation of Cloud Computing Environments and Evaluation of Resource Provisioning Algorithms. Software: Practice and Experience (SPE), 41(1):23-50, ISSN: 0038-0644, Wiley Press, New York, USA, January 2011.

9. Cardosa M, Korupolu MR, Singh A. Shares and utilities based power consolidation in virtualized server environments. In Proceedings of the 11th IFIP/IEEE International Symposium on Integrated Network Management. USA, June 2009.

10. Chase JS, Anderson DC, Thakar PN, Vahdat AM, Doyle RP. Managing energy and server resources in hosting centers. In Proceedings of the 8th ACM Symposium on Operating Systems Principles. Banff, Canada, October 2001.

11. Feng XA, Mok AK. A model of hierarchical real-time virtual resources. In Proceedings of the 23rd IEEE Real-Time Systems Symposium. Austin, USA, December 2002.

12. Gandhi A, Harchol-Balter M, Das R, Lefurgy C. Optimal power allocation in server farms. In Proceedings of the 11th International Joint Conference on Measurement and Modeling of Computer Systems. Seattle, USA, June 2009.

13. Ge R, Feng X, Cameron KW. Performance-constrained distributed DVS scheduling for scientific applications on power-aware clusters. In Proceedings of the ACM/IEEE Supercomputing Conference. Seattle, USA, November 2005.

14. Hsu C, Feng W. A power-aware run-time system for high-performance computing. In Proceedings of the ACM/IEEE Supercomputing Conference. Seattle, USA, November 2005. 
15. Kappiah N, Freeh VW, Lowenthal DK. Just in time dynamic voltage scaling: Exploiting inter-node slack to save energy in MPI programs. In Proceedings of the ACM/IEEE Supercomputing Conference. Seattle, USA, November 2005.

16. Kim KH, Beloglazov A, Buyya R. Power-aware provisioning of cloud resources for real-time services. In Proceedings of the 7th International Workshop on Middleware for Grids, Clouds and e-Science (MGC 2009). Urbana Champaign, USA, December 2009.

17. Kim KH, Buyya R, Kim J. Power aware scheduling of bag-of-tasks applications with deadline constraints on DVS-enabled clusters. In Proceedings of the 7th IEEE International Symposium on Cluster Computing and the Grid (CCGrid'07). Rio de Janeiro, Brazil, May 2007.

18. Kusic D, Kephart JO, Hanson JE, Kandasamy N, Jiang G. Power and performance management of virtualized computing environments via lookahead control. In Proceedings of the 5th IEEE International Conference on Autonomic Computing (ICAC 2008). Chicago, USA, June 2008.

19. Markoff J, Lohr S. Intel's huge bet turns iffy. New York Times Technology Section, September 2002.

20. Niu L, Quan G. Reducing both dynamic and leakage energy consumption for hard real-time systems. In Proceedings of the International Conference on Compilers, Architectures and Synthesis for Embedded Systems (CASES 2004). Washington, DC, USA, September 2004.

21. Ongaro D, Cox A, Rixner S. Scheduling i/o in virtual machine monitors. In Proceedings of the 4 th $A C M$ SIGPLAN/SIGOPS International Conference on Virtual Execution Environments. Seattle, USA, March 2008.

22. Rusu C, Ferreira A, Scordino C, Watson A, Melhem R, Mosse D. Energy-efficient real-time heterogeneous server clusters. In Proceedings of the 12th IEEE Real-Time and Embedded Technology and Applications Symposium. San Jose, USA, April 2006.

23. Scheihing P. Creating energy efficient data centers. In Data Center Facilities and Engineering Conference. Washington, DC, USA, May 2007.

24. Shin I, Lee I. Compositional real-time scheduling framework with periodic model. ACM Transactions on Embedded Computing Systems, 7(3), April 2008.

25. Son SW, Malkowski K, Chen G, Kandemir M, Raghavan P. Integrated link/cpu voltage scaling for reducing energy consumption of parallel sparse matrix applications. In Proceedings of the 20th IEEE International Parallel and Distributed Processing Symposium. Greece, April 2006.

26. Srikantaiah S, Kansal A, Zhao F. Energy aware consolidation for cloud computing. In Proceedings of the Workshop on Power Aware Computing and Systems (HotPower 2008). San Diego, USA, December 2008.

27. Verma A, Ahuja P, Neogi A. pMapper: Power and migration cost aware application placement in virtualized systems. In Proceedings of the 9th ACM/IFIP/USENIX International Conference on Middleware. Leuven, Belgium, December 2008.

28. Verma A, Ahuja P, Neogi A. Power-aware dynamic placement of HPC applications. In Proceedings of the 22nd ACM International Conference on Supercomputing (ICS 2008). Agean Sea, Greece, June 2008.

29. VirtualLogicx Real-Time Virutulalization and VLX. VirtualLogix, http://www.osware.com.

30. Wang L, Lu Y. Efficient power management of heterogeneous soft real-time clusters. In Proceedings of the 29th IEEE Real-Time Systems Symposium. Barcelona, Spain, December 2008.

31. Warren W, Weigle E, Feng W. High-density computing: A 240-node Beowulf in one cubic meter. In Proceedings of the ACM/IEEE Supercomputing Conference. Baltimore, USA, November 2002.

32. Yoo S, Park M, Yoo C. A step to support real-time in a virtual machine monitor. In Proceedings of the 6th IEEE Consumer Communications and Networking Conference. Las Vegas, USA, January 2009. 